The most often-mentioned method to flatten 0-rings is boiling. This method works well with viton. The advantages of boiling are that the method is easy to do reproducibly, and it drives out a lot of 'glup,' to use the technical term. The disadvantage is that the water must then be removed. For viton, this is pretty easy, since that elastomer can be baked out at about $200^{\circ} \mathrm{C}$.

Another method is heating to $50^{\circ} \mathrm{C}$ in an oven. This is not quite high enough for viton, which will not lie flat after an overnight heating at that temperature. Heating at a somewhat higher temperature should work. The advantages of the oven method are that one need not remove water, and it is the best-controlled method for those elastomers, such as buna- $\mathrm{N}$ and fluorocarbons, that cannot be heated above $70^{\circ} \mathrm{C}$.

Following the heating process, the 0 -ring can be cooled either slowly or rapidly. Slow cooling works, but there may be situations where shock cooling would be advantageous. In particular, to shape an 0-ring to a particular non-flat or non-round configuration, it might be good to heat, shape, then shock-cool.

Another idea is to put the 0-ring around a beaker and freeze it in the round state. This method can work if condensation is avoided, but it depends on where the 0-ring goes. If it has to fit on the bottom of a part, such as a column section, the reassembly of the column takes so long that the 0-ring would thaw and fall out.

A caution about silicone 0 -rings is that they are very permeable to $\mathrm{He}$. As a result, leak-checking can give false positives for several days.

Both viton and silicone 0 -rings can be baked out at about $200^{\circ} \mathrm{C}$, and that may be a good idea for a standard practice, since it will drive off volatiles in the 0 -rings. Buna- $\mathrm{N}$ and fluorocarbon cannot be heated above $70^{\circ} \mathrm{C}$, and at that for only a few hours. Buna-N just melts, but fluorocarbon decomposes. Ethylene-propylene is the most radiation-resistant of the common elastomers. It is used for seals that are close to the beam, and it remains relatively flexible under circumstances where either viton or neoprene harden. The Parker 0-Ring Handbook* is a useful source of information about many properties and applications of various available elastomers.

Since elastomers are treated by crosslinking and with additives, and are, no doubt, optimized for particular applications, the appropriate temperatures and times for particular treatments should be determined experimentally, rather than relying on information from books. Some information, such as decomposition temperatures, can be obtained reliably from books and used to set upper limits for trial runs, but even in this case, it is probably better to talk to the manufacturer before approaching these limits, since the treatments may significantly change them

\section{Bill Tivol,}

\section{Wadsworth Center and Biological Microscopy} and Image Reconstruction Resource

Summarized by Bill Tivol from his own information and others responses to a question asked by Dr. Tivol on the MSA microscopy mail server.

* Parker Seal Group

0-Ring Division

2360 Palumbo Drive

P.O. Box 11751

Lexington KY 40512

\section{Preventing Embedding Resin Bubbles}

I find that I can eliminate bubble formation in polymerizing blocks of resin by making sure I preheat the molds (capsules, flat molds, whatever) AND the labels in a $60^{\circ} \mathrm{C}$ oven for half a day to overnight before transferring the sample and resin to them. This seems to dry out and/or degas these elements, and I never get bubbles. (Never again will I try to de-bubble by pulling a vacuum on my resin. I HATE cleaning up the mess if it burps!)

Tina (Weatherby) Carval ho, University of Hawaii
I have had some success reducing the vibration evident on my SEM by making four pads from Sorbothane. I use a fairly stiff number of Sorbothane about $3 / 8$ inch $(15 \mathrm{~mm})$ thick aniu cut four pads about four inches $(100 \mathrm{~mm})$ square. Make eight steel plates to go on top and bottom of the Sorbothane pads, making steel-and-Sorbothane sandwiches. Put these under each corner of the column section.

The vibration on my SEM (on the fourth floor) went down about by half.

\section{Mary Mager, University of British Columbia}

I would like to add that one of the secrets to vibration reduction is mass - the greater the mass, the less the vibration (thus the heavy weights on rotary pump hoses). A relatively cheap and simple way to add mass to your microscope column is to place it on a concrete slab. These can be bought at home supply stores or made on site. Buy a bag of concrete, build a plywood tray, pour in the concrete and let it set. The form does not have to be removed except for aesthetic reasons, and denser weights (scrap iron, lead bricks) can be placed in the form before pouring to add mass.

The concrete slab works best if placed on a cushion of some kind. such as Mager's Sorbothane pads. With the greater mass, you may want to use bigger pads, or more of them.

It is easiest to handle the slab if the pads are placed where the column is wanted, the form placed on top of them, and then the concrete is poured. After it cures, the microscope or microscope column is placed on the slab.

Caveat: greater masses also have lower resonant frequencies, and a column's suspension may be tuned to pass certain, possibly low, frequencies.

A mass that matches this frequency may cause resonance problems. The column manufacturer can be consulted to find which frequencies the suspension passes.

\section{Phil Oshel}

\section{Making a High-Resolution Standard for SEM}

If you have a sputter coater or evaporator, you can make your own high-resolution standard. I've had good luck making calibration standards like this (I use them for camera constant calculation, resolution, demos, etc., in TEM). It's good practice in thin film making for students, etc.

1) Make a thin carbon film by carbon coating a formvar, butvar, etc coated grid. How thin the carbon should be depends on the final use if you want to see the gold lattice in TEM it should be painfully thin.

2) Remove the plastic backing by placing it in a (glass) Petri dish lined with a filter paper pad soaked in chloroform (in a fume hood).

3) After an overnight stint in the Petri dish, you should have mostly carbon left on the grids. Place them in your sputter coater and give them a short blast of Au. A pure gold target is best; if you use Au/Pd in yours, I'm not sure what you'll get. About $1 / 12$ th of the normal time needed to coat an average SEM sample should work.

4) You will have a collection of isolated islets of Au. Carbon tape it to a stub and have a look.

John Heckman, Michigan State University

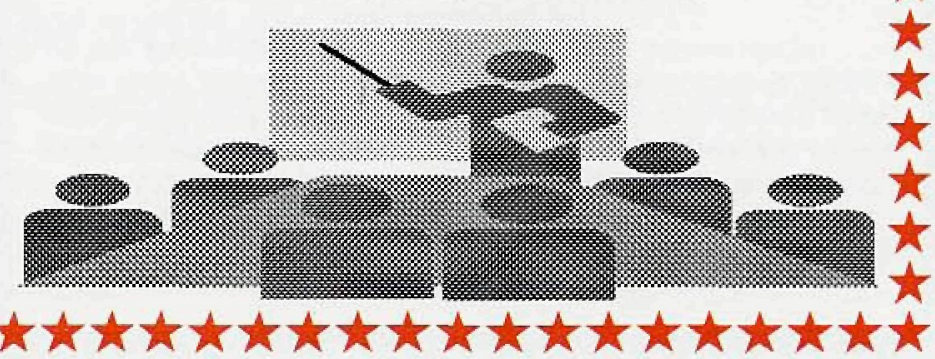

